\title{
Ultrafast Electron Diffraction for the Dynamical Study of 2D Materials
}

\author{
A. A. Petruk ${ }^{1}$, Nicolás Rivas ${ }^{1}$, T. Dekker ${ }^{2}$, K. Pichugin ${ }^{1}$ A. W. Tsen ${ }^{3}$ and G. Sciaini ${ }^{1}$ \\ ${ }^{1 .}$ Ultrafast electron Imaging Laboratory (UeIL), Department of Chemistry, and Waterloo Institute for \\ Nanotechnology (WIN), University of Waterloo, Waterloo, Canada. \\ 2. Department of Electrical and Computer Engineering (ECE), and Institute for Quantum Computing \\ (IQC), University of Waterloo, Waterloo, Canada. \\ 3. Department of Chemistry, Institute for Quantum Computing (IQC), and Waterloo Institute for \\ Nanotechnology (WIN), University of Waterloo, Waterloo, Canada.
}

The observation of atomic rearrangements as they occur during phase transformations and chemical reactions has been the exclusive domain of computer simulations. Advances in the generation of ultrashort structural probes over the last thirty years have finally brought such "molecular views" to the experimental ground. The challenge has been the creation of hard X-ray or electron pulses bright and short enough to capture atoms on the fly; i.e. sub-picosecond pulses and the shorter the better to avoid motion blur in stroboscopically recorded images. The brightest ultrafast source of hard X-ray on earth (LCLS, Stanford) is now delivering pulses as short as 5 femtoseconds $\left(1 \mathrm{fs}=10^{-15} \mathrm{~s}\right)$ with enough X-ray photons to attain high-quality diffraction in a single shot [1]. On the other hand, laboratory-scale femtosecond electron diffraction (FED) setups have also emerged as a powerful means to monitor ultrafast structural dynamics. Electron pulse compression schemes [2-4] have greatly improved and can provide nowadays a temporal resolution of $100 \mathrm{fs}$ [5]. Furthermore, new all-electrostatic electron sources are also expected to deliver soon multi-electron bursts with less than $30 \mathrm{fs}$ in length at fullwidth-half-maximum [6].

Electrons moving at kinetic energies in the range of 30 to $300 \mathrm{keV}$ have a cross section which is $\sim 10^{4}$ to $10^{5}$ times higher than $10-\mathrm{keV}$ X-rays, a relative measure of their scattering efficiency. Their mean-freepath of 10 to $100 \mathrm{~nm}$ makes electrons particularly sensitive to the nanoscale and also matches the typical optical penetration depth of the excitation laser pulse. Therefore, well-defined photoexcited carrier densities are easily obtained. In addition, the very small de Broglie wavelength (quasi-flat Ewald sphere) of keV electrons makes them uniquely qualified for monitoring in-plane atomic displacements. Electron diffraction is known to capture the entire 2D Brillouin zone at once. This fact greatly simplifies the observation and distinction among different dynamical processes that give rise to changes in the diffraction intensities [7, 8]. With a temporal resolution of about $100 \mathrm{fs}$ and a sensitivity better than 0.01 $\AA$ [7], the proposed methodology will bring new vistas in cooperative many body phenomena governing the electronic properties of $2 \mathrm{D}$ quantum materials.

The development of a novel 300-kV FED setup, based on an all-electrostatic femtosecond electron source alongside results obtained for a 2D transition metal dichalcogenide (TMDC), $\mathrm{MoTe}_{2}[8,9]$ will be presented. The FED instrument is shown in figure 1 and is driven by a femtosecond (fs) laser (Pharos, Light Conversion). The FED setup comprises a custom designed high vacuum chamber, a homemade $300-\mathrm{kV}$ high voltage feedthrough, a large-area and fast readout detection camera (Andor, Zila 5.5), vacuum pumps, and motorized stages with sub-micron precision (attocube) for sample positioning. The general FED methodology follows; ultrashort electron pulses are generated via photoemission off a gold photocathode. The cathode head is held at negative voltage, typically between $100 \mathrm{kV}$ and $300 \mathrm{kV}$ with respect to a ground anode plate. The electron bursts, accelerated by a DC electric field, exit the anode 
aperture to scatter off the sample and generate a diffraction pattern on a phosphor screen. A large-area CMOS detector finally records a series of diffraction patterns that are taken at different time delays between the electron probe pulse and excitation pulse in order to reconstruct the time-dependent structural changes. We found that the combination of femtosecond transient absorption and FED experiments provides a detailed view of electron-phonon and phonon-phonon relaxation pathways in 2D quantum materials [10].

References:

[1] C. Gutt et al, Phys. Rev. Lett. 108 (2012), p. 024801.

[2] van T. Oudheusden et al, Phys. Rev. Lett. 105 (2010), p. 264801.

[3] M. Gao et al, Nature 496 (2013), p. 343.

[4] V. R. Morrison et al, Science 346, (2014), p. 445.

[5] M. R. Otto et al, Struct. Dyn. 5 (2017), p. 051101.

[6] A. Petruk et al, Struct. Dyn. 4 (2017), p. 044005.

[7] M Eichberger et al, Nature 468, (2010), p. 799.

[8] M. Chhowalla et al, Nat. Chem. 5 (2013), p. 263.

[9] A. W. Tsen et al, Proc. Nat. Acad. Sci. 112 (2015), p. 15054.

[10] The authors acknowledge funding from the Natural Sciences and Engineering Research Council of Canada. GS acknowledges the funding provided by the Canada Foundation for Innovation.

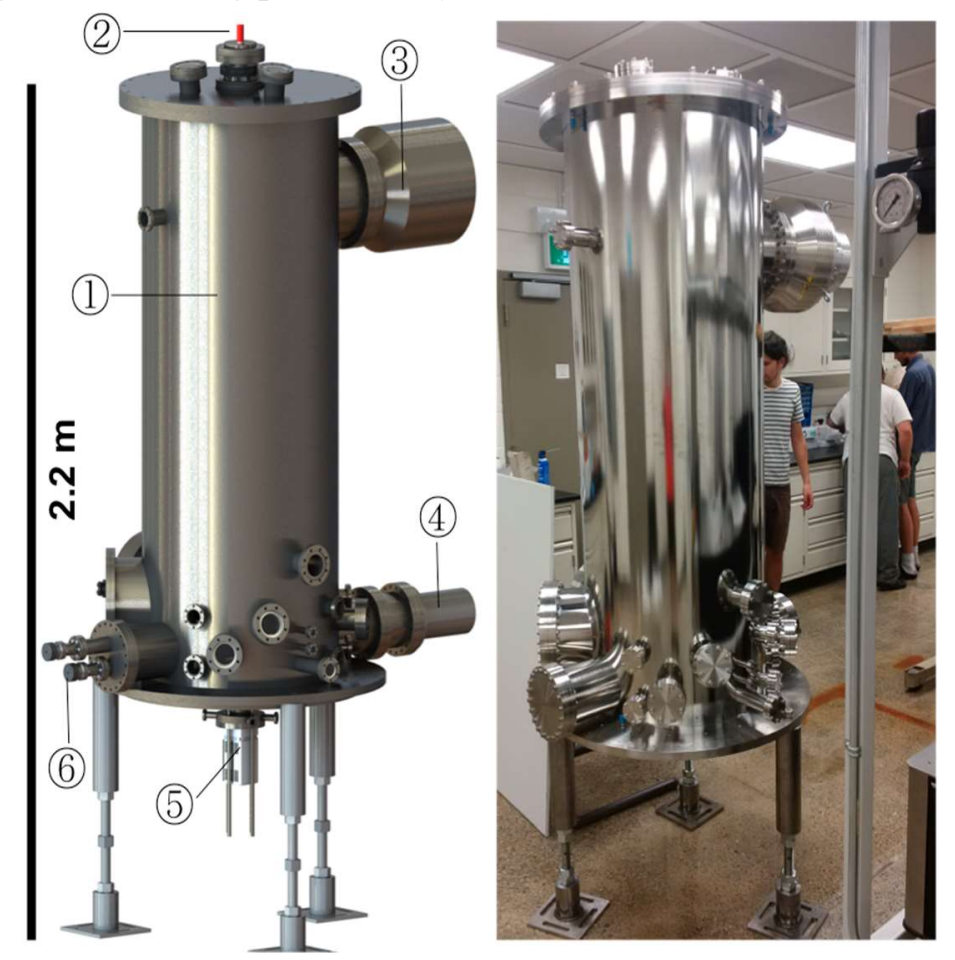

Figure 1. CAD design (left) and installed 300-kV FED setup (right). Shown components: (1) vacuum chamber (background pressure $\sim 10^{-8}$ mbar); (2) high voltage cable; (3) turbo molecular pump; (4) Helium cryohead with vibration damper; (5) large area 2D CMOS detector; (6) linear manual manipulators for apertures and coarse positioning of motorized sample stages. 